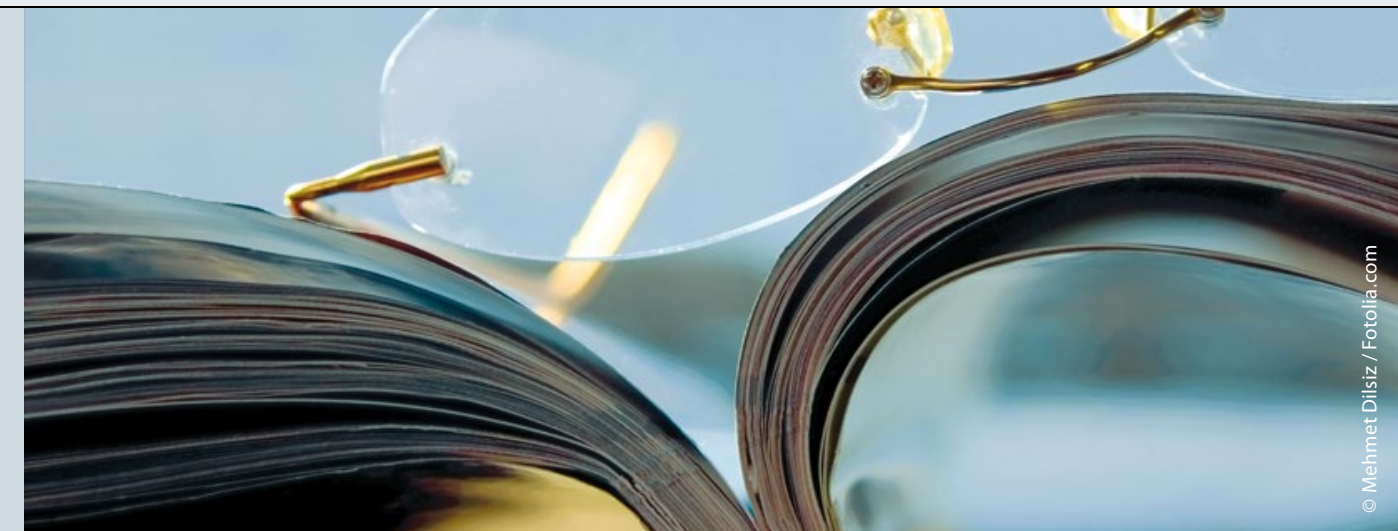

\section{Wie lässt sich die primäre Negativsymptomatik behandeln?}

\author{
Eine Metaanalyse untersuchte den Effekt pharmakologischer und \\ psychologischer Interventionen auf die primäre Negativsymptomatik.
}

$\mathrm{D}$ ie Schizophrenie stellt ein heterogenes klinisches Syndrom dar. Die klassische Einteilung der Symptomatik unterscheidet die Positiv- von der Negativsymptomatik. Erstere ist charakterisiert als ein „Zuviel“ (z. B. Wahninhalte, Halluzinationen), die zweite als ein „Zu-

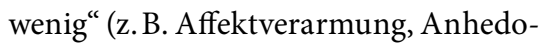
nie, Antriebsarmut). Die Negativsymptomatik stellt zusammen mit den kognitiven Störungen den Hauptgrund für die massive funktionelle Einschränkung vieler schizophrener Patienten dar und bildet so die Hauptursache für die enormen Kosten dieser Erkrankung für die Krankenkassen, aber auch für das Gemeinwesen insgesamt (beispielsweise durch die hohe Zahl an Frühberentungen).

Während es klare Richtlinien für die Behandlung der sogenannten sekundä- ren Negativsymptomatik (also durch antipsychotische Pharmakotherapie oder die Positivsymptomatik hervorgerufen) gibt (z. B. Reduktion/Absetzen sedierender Begleitmedikation, keine zu hohe Dosis von starken D2-Blockern, effektive Therapie von begleitender Angstsymptomatik), ist unklar, wie die primäre Negativsymptomatik behandelt werden soll.

Dieser Frage hat sich die Metaanalyse von Fusar-Poli gewidmet. Eingeschlossen wurden 168 placebokontrollierte Studien, deren primäres Ziel es war, die Effektivität von pharmakologischen und psychologischen Interventionen auf primäre $\mathrm{Ne}$ gativsymptomatik zu untersuchen. Insgesamt wurden so 6.503 Patienten, die eine Intervention, und 5.815 Patienten, die Placebo erhielten, berücksichtigt. Es wurde kein Publikationsbias festgestellt. Die

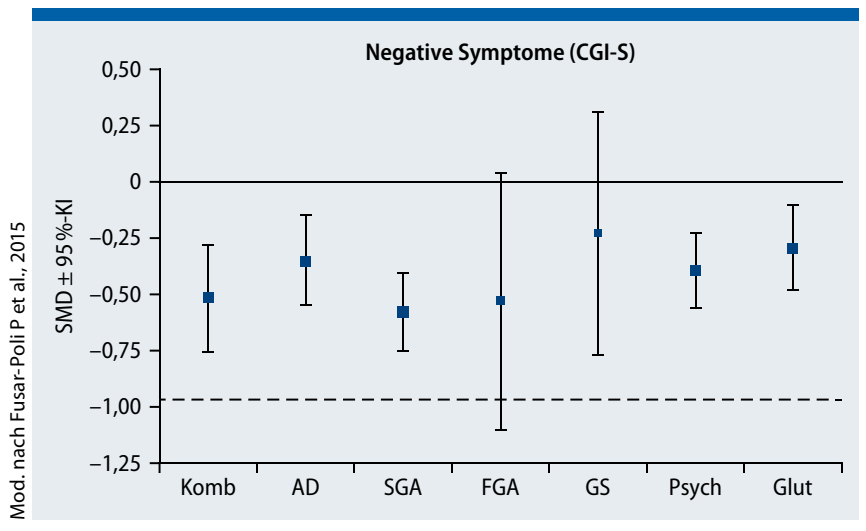

Effektstärken (95\%-KI) der unterschiedlichen Interventionsgruppen im Vergleich zu Placebo waren wie folgt (Abb. 1): Second generation Antipsychotika (SGA): - 0,579 $(-0,755 ;-0,404)$, Antidepressiva (AD): $-0,349(-0,551 ;-0,146)$; Kombinationen (Komb): $-0,518$ (-0,757; $-0,279)$; glutamaterge Pharmaka (Glut): $-0,289(-0,478 ;-0,1)$; psychologische Interventionen (Psych): $-0,396(-0,563$; $-0,229)$. Kein Effekt wurde für First generation Antipsychotika (FGA) mit - 0,531 $(-1,104 ; 0,041)$ und die Gehirnstimulation (GS) mit - $0,228(-0,775 ; 0,319)$ gefunden. Zwischen den einzelnen Gruppen (also auch nicht zwischen SGA und FGA) gab es keinen statistisch signifikanten Unterschied.

\section{Kommentar}

In dieser Untersuchung konnte der Effekt von Clozapin nicht bewertet werden, da die Autoren keine einzige Studie fanden, in denen Clozapin mit Placebo bei primärer Negativsymptomatik verglichen wurde.

Die Autoren stellen fest, dass es zwar einige statistisch signifikante Unterschiede bei dieser Metaanalyse gab, dass diese allerdings klinisch kaum relevant seien. Dieser pessimistischen Sicht kann ich mich nicht anschließen, markieren doch Effektstärken um 0,5 eindeutig einen mittleren Effekt, was bei der Zielvariable, nämlich der Behandlung der - wie bekannt - schlecht zu beeinflussenden primären Negativsymptomatik, meines Erachtens ein beachtenswertes Ergebnis darstellt und auch klinisch beachtet werden sollte.

Prof. Dr. med. Hans-Peter Volz

Fusar-Poli $\mathrm{P}$, et al. Treatments of negative symptoms in schizophrenia: meta-analysis of 168 randomized placebo-controlled trials. Schizophr Bull 2015; 41: 892-99 